ISSN 2215-3535

\title{
Estereotipos sobre la población adulta mayor en tres grupos etarios de cuidadores de personas mayores dependientes
}

\section{Stereotypes About the Elderly Adult Population in Three Age Groups of Carers of Dependent Elderly People}

\author{
Daysy Katherine Pabón Poches ${ }^{1}$ \\ Angie Lorena Flórez García ${ }^{2}$ \\ Lina Marcela Sanabria Vera ${ }^{3}$ \\ 1,2,3 Universidad de Investigación y Desarrollo-UDI \\ Seccional Bucaramanga, Colombia
}

Resumen. La presencia de estereotipos en cuidadores implica efectos desfavorables para las personas adultas mayores dependientes. Objetivo. Este estudio de carácter cualitativo fenomenológico se planteó como propósito describir los estereotipos que existen hacía los adultos mayores en cuidadores de tres grupos etarios (adultos jóvenes, medios y mayores). Método. Se aplicó una entrevista semi-estructurada a 20 cuidadores de personas mayores dependientes, el reporte se analizó por medio de codificación axial utilizando las categorías de estereotipos biológicos, cronológicos, psicológicos y sociológicos. Resultados. Se identificaron estereotipos positivos y negativos en los tres grupos etarios, aunque predominan los negativos relacionados con aspectos biológicos como la pérdida y la enfermedad.

Palabras clave. Estereotipos, cuidador, vejez, envejecimiento.

Abstract. The presence of stereotypes in carers implies unfavourable effects for dependent elderly people. Objective. This qualitative phenomenological study was therefore intended to describe the stereotypes that exist towards elderly adults in caregivers of three age groups (young, middle and older adults). Method. A semistructured interview was applied to 20 caregivers of dependent older adults. The report was analyzed via axial coding using biological, chronological, psychological and sociological stereotype categories. Results. Positive and negative stereotypes were identified in the three age groups, although negative stereotypes related to biological aspects such as loss and disease prevail.

Keywords. Stereotypes, caregiver, old age, aging.

${ }^{1}$ Daysy Katherine Pabón Poches. Universidad de Investigación y Desarrollo - UDI, Seccional Bucaramanga, Colombia. Dirección postal: Calle 9 No. 23-55, Santander, Colombia. E-mail: dpabon1@udi.edu.co

${ }^{2}$ Angie Lorena Flórez García. Universidad de Investigación y Desarrollo - UDI, Seccional Bucaramanga, Colombia. E-mail: alorenaflorez@gmail.com ${ }^{3}$ Lina Marcela Sanabria Vera. Universidad de Investigación y Desarrollo - UDI, Seccional Bucaramanga, Colombia. E-mail: Imarcelasanabria@gmail.com 


\section{Introducción}

Los estereotipos son creencias despectivas sobre grupos sociales en un sistema caracterizado por la separación del individuo dentro de roles, clases, posiciones o estatus. Tienen como característica esencial la inexactitud de la información que contienen, puesto que permiten inferir cualidades idénticas para todos los miembros de un grupo; a través de estas creencias sociales, los individuos se uniformizan y se los priva de su identidad (Agredo, 2010; Organización Mundial de la Salud [OMS], 2015; Sánchez \& Triana, 2009).

Por otro lado, Menéndez, Cuevas, Pérez y Lorence (2016) afirman que los estereotipos son ideas apriorísticas, sesgadas y no contrastadas (o contrastadas solo parcialmente) con la realidad, hacia una persona en razón a su pertenencia a un colectivo con características supuestamente homogéneas. Se ha considerado tradicionalmente que las imágenes negativas y peyorativas forman el contenido de los estereotipos; sin embargo, en algunos estudios se indica que los miembros de un mismo grupo pueden soportar simultáneamente estereotipos tanto positivos como negativos (Agredo, 2010; OMS, 2015; Sánchez \& Triana, 2009).

Uno de los grupos sociales que se ha visto impactado por la presencia de estereotipos es el de las personas adultas mayores (en adelante, PAM). Ubicados en la etapa denominada vejez, la cual se da a partir de los 60 años (Ministerio de Salud de Colombia, 2016) y hace parte del proceso de envejecimiento. El envejecimiento incluye modificaciones morfológicas y fisiológicas que aparecen desde el momento del nacimiento; es un fenómeno irreversible que afecta a todos los seres vivos, aunque de manera diferente o con distinta intensidad, es variable y diferencial, y no uniforme u homogéneo (Ballesteros, 2007; Dulcey \& Uribe, 2002). Este proceso está condicionado por factores intrínsecos y extrínsecos, como el estilo de vida y los hábitos, así como aspectos biológicos, sociales y psicológicos que se dan dentro de la interacción de la herencia y el ambiente (Ballesteros, 2007; Hoyl-Moreno, 2016).

Según Agredo (2010), entre las creencias estereotipadas más comunes sobre las PAM se encuentran las relacionadas con la etapa de la vejez y el proceso de envejecimiento. Tales como:

... las personas mayores están socialmente aisladas; las personas mayores están enfermas, son frágiles y dependen de otras personas; la mayoría de las personas mayores tienen algún grado de deterioro cognitivo; las personas mayores están deprimidas; las personas mayores se vuelven difíciles de tratar y son, con el paso de los años, más rígidas; las personas mayores raramente se enfrentan a los declives inevitables asociados con el envejecimiento (p. 274).

En la actualidad existen teorías que se oponen a la perspectiva de pérdida y deterioro asumida propia de la vejez o del proceso de envejecimiento, y que sustenta la base de los estereotipos hacia las PAM. Tal como la perspectiva del ciclo vital, la cual facilita una noción de vejez y envejecimiento que trata de integrar una visión multidimensional, multidireccional y multicausal; lo que permite entender las vulnerabilidades y oportunidades que las personas acumulan a lo largo de la vida. Afirma además, la posibilidad de mejorar el uso de recursos escasos, facilitando la identificación de riesgos, brechas y la priorización de intervenciones claves (Dulcey \& Uribe, 2002; Ministerio de Salud de Colombia, 2016). 
Las PAM han adquirido una connotación negativa que los afecta no solo a sí mismos, sino a personas en etapas más tempranas. Pues genera un efecto cascada, ya que las ideas o creencias asociadas a la vejez o el envejecimiento que presentan personas jóvenes o de mediana edad, también les afectará en su vejez, en su proceso de envejecimiento y en la interacción que se genere con la población PAM (Dulcey \& Uribe, 2002).

Sobre la interacción con las PAM, este estudio resalta la relación de cuidado - dependencia. Cada vez más visible debido al aumento de la proporción y número de PAM en las poblaciones de todo el mundo (OMS, 2018). Así como el aumento de la cantidad de familiares o profesionales que se hacen cargo de los cuidados de estos (Espín, 2008; Cerquera, Pabón, Granada, \& Galvis, 2016; Pabón, Galvis, \& Cerquera, 2014).

Cuidador/paciente dependiente integran una diada relacional que surge como una opción de desarrollo humano (Sánchez, Chaparro, \& Carrillo, 2016); la diada se debe entender como un sujeto de atención en sí mismo (Chaparro, Sánchez, \& Carrillo, 2014). Se ha demostrado que el cuidador puede verse afectado por una serie de consecuencias negativas de carácter físico, emocional, psicológico y/o social, lo que a su vez puede afectar a la persona dependiente, llegando incluso a ser víctima de negligencia y otros tipos de maltrato (Pabón \& Delgado, 2017; Vicente-Ruiz, De la Cruz, Morales, Martínez, \& Villareal, 2014).

El cuidador es la persona que asiste o cuida a otra afectada por cualquier tipo de discapacidad, minusvalía o incapacidad que le dificulta o impide el desarrollo normal de sus actividades vitales o de sus relaciones (Flóres, Rivas, \& Seguel, 2012; Lavrestky, 2005). Estos pueden clasificarse en cuidadores formales e informales; los cuidadores formales se caracterizarán por recibir una remuneración económica, tener un horario específico de dedicación y no tener ningún vínculo consanguíneo con la PAM dependiente; el cuidador informal no recibe pago, convive con la PAM dependiente y tiene un vínculo consanguíneo (Aparicio, 2018, citado en Pabón et al., 2014).

Lo anteriormente expuesto, sustenta la necesidad de realizar una aproximación al estudio de los estereotipos que pueden presentar los cuidadores de PAM dependientes, ya que conocerlos contribuye al abordaje de una diada que resulta compleja. Pero también ineludible para aportar a una visión holística, especialmente ante una realidad de cuidado que ha generado modificaciones y efectos dentro de las familias (Chaparro et al., 2014), y sobre diferentes grupos etarios. Para ello, se tendrá en cuenta la propuesta de clasificación de estereotipos de Rodríguez-Domínguez (1989) expuesta en la Tabla 1, utilizada en diversas investigaciones (Carbajo, 2009; Fernández \& Antón, 2018).

Para indagar sobre la presencia de estereotipos es necesario priorizar la experiencia y los significados de los participantes; por lo que se requiere una aproximación y análisis basados en el diseño fenomenológico (Hernández, Fernández, \& Baptista, 2014). Este permite al investigador realizar preguntas dirigidas a la comprensión del significado que las experiencias vividas tienen para los participantes del estudio, seleccionar las palabras o frases que describen particularidades de la experiencia estudiada, agruparlos y organizarlos (Álvarez-Gayou, 2003).

Algunos estudios sobre estereotipos se han realizado mayormente en dos grupos etarios: PAM (Callís-Fernández, 2011; Sánchez, Trianes, \& Mena, 2009) y jóvenes (Arnorld- 
Tabla 1

Clasificación de estereotipos

\begin{tabular}{|c|c|c|}
\hline Estereotipo & Descripción & Efecto \\
\hline Cronológico & $\begin{array}{l}\text { Acentúa el criterio de normas de } \\
\text { edad y equipara el envejecimiento } \\
\text { con el número de años vividos. }\end{array}$ & $\begin{array}{l}\text { Produce una discriminación por la edad } \\
\text { que puede impedir a las personas mayores } \\
\text { alcanzar la felicidad y productividad total. }\end{array}$ \\
\hline Biológico & $\begin{array}{l}\text { Consideración médica sobre la vejez } \\
\text { que ha incidido en la concepción del } \\
\text { envejecimiento como involución y } \\
\text { senilidad. }\end{array}$ & $\begin{array}{l}\text { Ocasiona una carga de achaques físicos, } \\
\text { abundancia de enfermedades, trastornos } \\
\text { psicofisiológicos, que crea la necesidad } \\
\text { de asistir permanentemente a hospitales } \\
\text { y geriátricos desde la perspectiva de la } \\
\text { cercanía de la muerte. }\end{array}$ \\
\hline Psicológico & $\begin{array}{l}\text { Se ha acentuado el concepto } \\
\text { de deterioro y declive de los } \\
\text { recursos psicológicos sensoriales, } \\
\text { atencionales, memorísticos, } \\
\text { cognoscitivos, aptitudinales o de } \\
\text { habilidades, de personalidad, de } \\
\text { carácter, etc. }\end{array}$ & $\begin{array}{l}\text { Conlleva a una escasa o nula creatividad, } \\
\text { aislamiento intimista, ansiedad, depresiones, } \\
\text { comportamientos rígidos e inflexibles y } \\
\text { cambios de humor. Sin dejar de lado que } \\
\text { en esta etapa puede encontrarse personas } \\
\text { mayores creativas, activas y bien adaptadas } \\
\text { personal y socialmente. }\end{array}$ \\
\hline Sociológico & $\begin{array}{l}\text { Ha incidido tradicionalmente en } \\
\text { las connotaciones de inutilidad, } \\
\text { aislamiento, improductividad y } \\
\text { desvinculación de la persona mayor } \\
\text { respecto a los intereses sociales y } \\
\text { relacionales comunitarios. }\end{array}$ & $\begin{array}{l}\text { Lleva a la equiparación de la vejez como la } \\
\text { etapa de jubilación. Uno de los estereotipos } \\
\text { negativos más comunes con respecto a este } \\
\text { ciclo vital; enmarcado en la pobreza, asilo, } \\
\text { abandono y soledad. }\end{array}$ \\
\hline
\end{tabular}

Nota. Tomado de Rodríguez-Domínguez (1989, citado por Carbajo, 2009).

Cathalifaud, Thumala, Urquiza, \& Ojega, 2007; Cerquera, Meléndez, \& Villabona, 2012; Aristizabal, Moreno, Salas, \& Torres, 2009); en ambos indican percepciones estereotipadas. Además, se han realizado estudios que resaltan la importancia de comparar las percepciones por grupos etarios; con jóvenes y en adultos mayores de diez países de Latinoamérica (Lasagni et al., 2012) con niños, adolescentes, adultos jóvenes y adultos en mediana edad (Cerquera, Álvarez, \& Saavedra, 2010).

En cuanto a estudios que han explorado estereotipos en personas que mantienen interacción constante con las PAM, se encuentra el realizado con personal de salud de un hospital de la ciudad de Querétaro, México (Franco et al., 2010), profesionales de atención a PAM (Bustillo-López \& Fernández-Ballesteros, 2012; Castellano, 2014). En los que se ha confirmado que los estereotipos influyen de manera negativa sobre el bienestar de las PAM, arrojando evidencia de un desmejoramiento en la calidad de vida, el bienestar y un menor nivel de actividad general (Castellano, 2014). Lo que reitera la necesidad de indagar el fenómeno de los estereotipos en cuidadores; y evidencia que aún hay escaso reporte, especialmente en lo que experimentan los cuidadores informales. 


\section{Método}

\section{Diseño}

La presente investigación se ubica dentro del enfoque cualitativo y es de diseño fenomenológico, este diseño permite que el investigador se enfoque en la experiencia compartida de los participantes (Hernández-Sampieri et al., 2014). Lo que permite identificar estereotipos hacia las PAM entre cuidadores(as) de tres grupos etarios, a partir de la interpretación y análisis del discurso de estos. Además, se incluyeron informantes clave, los cuáles fueron seleccionados por la técnica bola de nieve.

\section{Participantes}

Este estudio incluye 20 participantes, 4 hombres y 16 mujeres, donde 2 de ellos son cuidadores formales y 18 son cuidadores informales. Estos se consideraron suficientes para obtener la comprensión del fenómeno de interés, debido a que se consiguió la saturación de las categorías propuestas, tal como lo recomienda Hernández-Sampieri et al. (2014).

Los participantes se distribuían en tres grupos conformados de la siguiente manera: 9 adultos jóvenes con edades entre 20 y 39 años, 6 adultos de mediana edad entre 41 y 59 años, y finalmente 5 adultos mayores con una edad de 60 años o más; todos con experiencias de cuidado de población adulta mayor dependiente dentro de municipio de Bucaramanga. Para su inclusión se consideró fundamental que el cuidador tuviese mínimo cinco horas de dedicación al cuidado del AM al día, por un mínimo seis meses; así como estar de acuerdo en participar de forma voluntaria, para lo cual se utilizó el consentimiento informado.

\section{Instrumentos}

Entrevista semiestructurada de estereotipos sobre la población adulta mayor: esta entrevista está conformada por 10 preguntas abiertas organizadas en cuatro categorías deductivas: estereotipos cronológicos, estereotipos biológicos, estereotipos psicológicos y estereotipos sociológicos, clasificación tomada de Carbajo (2009); consultar Tabla 2. Para obtener la versión final de la entrevista, descrita en la tabla 1 , se somete a un proceso de validación de contenido haciendo uso del método de evaluación por jueces, con juez único.

\section{Procedimiento}

Inicialmente se realiza un abordaje teórico que permite sentar las bases conceptuales y las ideas previas para la construcción y validación de contenido de la herramienta de recolección de información. Una vez definido el formato de la entrevista semiestructurada, se procedió a contactar a un experto, quien diligenciando la plantilla de evaluación propuesta por Pabón-Poches (2019) para validaciones de contenido, sugiere modificaciones que son acatadas y contrastadas por medio de una aplicación piloto a dos individuos.

En el proceso de aplicación, se solicitó la colaboración voluntaria de los participantes en la investigación, y se les solicitó información sobre otros cuidadores informales para hacer la búsqueda en bola de nieve. Seguidamente, se les informó sobre el interés fundamental de la investigación, sobre la grabación de un audio durante la entrevista; y finalmente, firmaron 
Tabla 2

Preguntas entrevista semiestructurada según categorias de análisis

\begin{tabular}{|c|c|c|c|}
\hline Estereotipo & Estereotipo & Estereotipo & Estereotipo \\
\hline Cronológico & Biológico & Psicológico & Sociológico \\
\hline $\begin{array}{l}\text { ¿A qué edad cree } \\
\text { usted que una } \\
\text { persona se convierte } \\
\text { en adulta mayor? }\end{array}$ & $\begin{array}{l}\text { ¿Qué piensa usted de } \\
\text { los cambios físicos } \\
\text { que se dan en la } \\
\text { adultez mayor? }\end{array}$ & $\begin{array}{l}¿ \text { Considera usted } \\
\text { que al llegar a ser } \\
\text { adulto mayor se } \\
\text { tienen cambios en el } \\
\text { estado de ánimo? }\end{array}$ & $\begin{array}{l}\text { Durante las diferentes } \\
\text { etapas de la vida, las } \\
\text { redes de apoyo son } \\
\text { importantes ¿qué } \\
\text { papel juegan estas en } \\
\text { la adultez mayor? }\end{array}$ \\
\hline \multirow[t]{2}{*}{$\begin{array}{l}\text { ¿Qué cambios se } \\
\text { pueden observar } \\
\text { en una persona que } \\
\text { usted considera } \\
\text { adulta mayor? }\end{array}$} & $\begin{array}{l}\text { ¿Con qué } \\
\text { características o } \\
\text { cambios físicos asocia } \\
\text { usted a una persona } \\
\text { adulta mayor? }\end{array}$ & $\begin{array}{l}\text { ¿Considera usted } \\
\text { que al llegar a ser } \\
\text { adulto mayor se } \\
\text { presentan cambios } \\
\text { en la personalidad? }\end{array}$ & $\begin{array}{l}\text { "Las personas mayores } \\
\text { son más confiables } \\
\text { que los jóvenes" } \\
\text { ¿qué piensa usted } \\
\text { de esa afirmación? }\end{array}$ \\
\hline & & $\begin{array}{l}\text { Dicen que con el } \\
\text { pasar de los años } \\
\text { las personas se } \\
\text { hacen más sabias } \\
\text { ¿Usted qué piensa? }\end{array}$ & $\begin{array}{l}\text { ¿Qué significado } \\
\text { tiene la muerte en } \\
\text { la adultez mayor? }\end{array}$ \\
\hline
\end{tabular}

el consentimiento informado. La recolección de datos se inicia con dos participantes considerados piloto, sus entrevistas hacen parte de la categorización inicial. Posterior a la aplicación piloto, se realizan cuatro entrevistas más y se refinan las categorías de análisis; así, el análisis preliminar permite identificar dos aspectos.

El primero es un criterio de inclusión, ya que, al identificar diferencias en el discurso a partir de la edad, se decide clasificar la muestra en tres grupos etarios. El segundo elemento, refiere a la similitud entre las experiencias de los participantes formales e informales, por lo que no se considera como criterio de inclusión el tipo de cuidador. Al momento de codificar y agrupar las categorías tras cuatro aplicaciones más, se ratifica la similitud entre las percepciones. Las categorías deductivas fueron cuatro y se mantienen hasta el final, surgen dos categorías emergentes y se incluyen seis subcategorías que contribuyen con la comprensión de las cuatro categorías iniciales.

Para asegurar el correcto funcionamiento de los participantes, se les administró de forma individual la entrevista semi-estructurada, asegurando condiciones mínimas de adecuación del contexto para la aplicación. Estas tuvieron una duración aproximada de 30 minutos y fueron realizadas en un período de dos semanas a un total de 20 cuidadores, número de participantes con los que se consiguió la saturación de las categorías.

La transcripción y codificación se realiza de forma paralela a la aplicación de las entrevistas; la codificación axial es realizada por dos de los investigadores y de forma independiente. Posteriora ello, se realiza un chequeo cruzado; finalmente se procede con un tercerinvestigador que asume el rol de auditor; este revisa y evalúa los análisis de ambos investigadores para terminar el proceso en conjunto, así se lleva a cabo la triangulación por auditoria. 


\section{Aspectos éticos}

Este proyecto de investigación se realiza bajo lineamientos del numeral 9 del artículo 2, de la Ley 1090 de 2006, la cual refiere aspectos relacionados a la investigación con humanos y al respeto de la dignidad y el bienestar de las personas que participan con pleno conocimiento de la investigación. Asimismo, se da rigor a lo indicado en el artículo 50, donde se hace énfasis sobre los principios éticos de respeto y dignidad, la importancia de salvaguardar el bienestar y los derechos de los participantes.

\section{Resultados}

A continuación, se describen las principales características de los participantes; después, se describen los estereotipos identificados en análisis del discurso. La Figura 1 permite evidenciar que la mayoría de los informantes se caracterizan por ser de género femenino (16), solteros (12) o casados (7), y brindan un cuidado informal (18). Generalmente, llevan desempeñándose como cuidadores entre 1 y 5 años (9), mientas que entre 11 y más de 21 años (6), dedicando un tiempo de cuidado durante el día mayormente de 1 a 5 horas (6) y de 6 a 10 horas (4).

El análisis del discurso de 20 informantes clave que comparten la experiencia de ser personas cuidadoras de PAM dependientes, permitió reconocer la presencia de estereotipos hacia la población que se encuentra a su cuidado; dichos estereotipos se agrupan en dos grandes categorías emergentes: estereotipos positivos y estereotipos negativos. Dentro de estos se ubican los cuatro tipos de estereotipos que corresponde a las categorías deductivos planteados inicialmente, el cronológico (CRO), Psicológico (PSI), Sociológico (SOC) y Biológico (BIO). Además, se encontraron ocho subcategorías que complementan el análisis de los estereotipos.

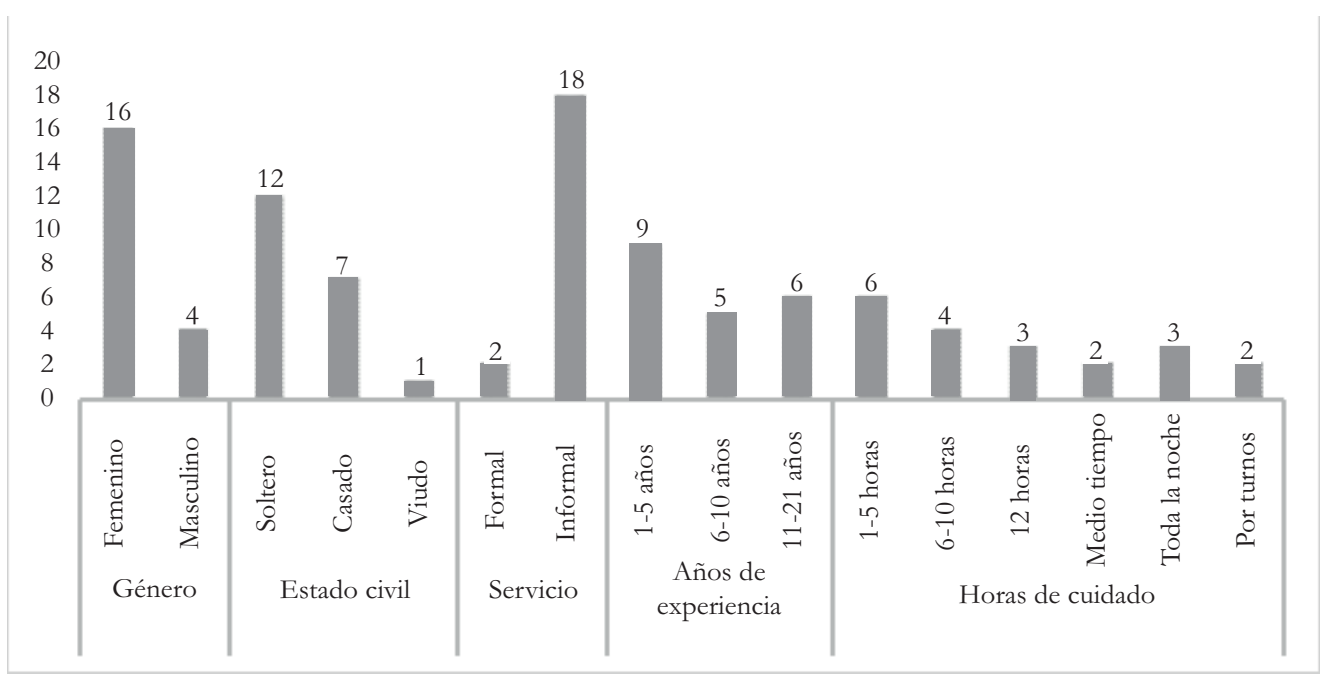

Figura 1. Descripción de los cuidadores 
Dentro de los estereotipos sociológicos se encuentran las subcategorías de apoyo social y rol social; dentro de la categoría de estereotipos psicológicos emergen las subcategorías de personalidad, emoción y experiencia; en la categoría de estereotipos cronológicos emergen las subcategorías cambios físicos y edad. Finalmente, en los estereotipos biológicos no surgen nuevas categorías y solo se encuentran estereotipos negativos al respecto, por lo que no se hallan estereotipos positivos relacionados con las consideraciones médicas de la vejez, como se observa en la Figura 2.

La Figura 3 permite visualizar que los estereotipos que muestran mayor manifestación son los de tipo negativos, al presentarse de forma equitativa dentro de las categorías CRO, PSI, SOC y BIO. Mientras que los estereotipos positivos solo se presentan en las categorías SOC, PSI y CRO con menor frecuencia, siendo los estereotipos SOC los más recurrentes dentro de los positivos y los estereotipos BIO los más recurrentes dentro de los negativos.

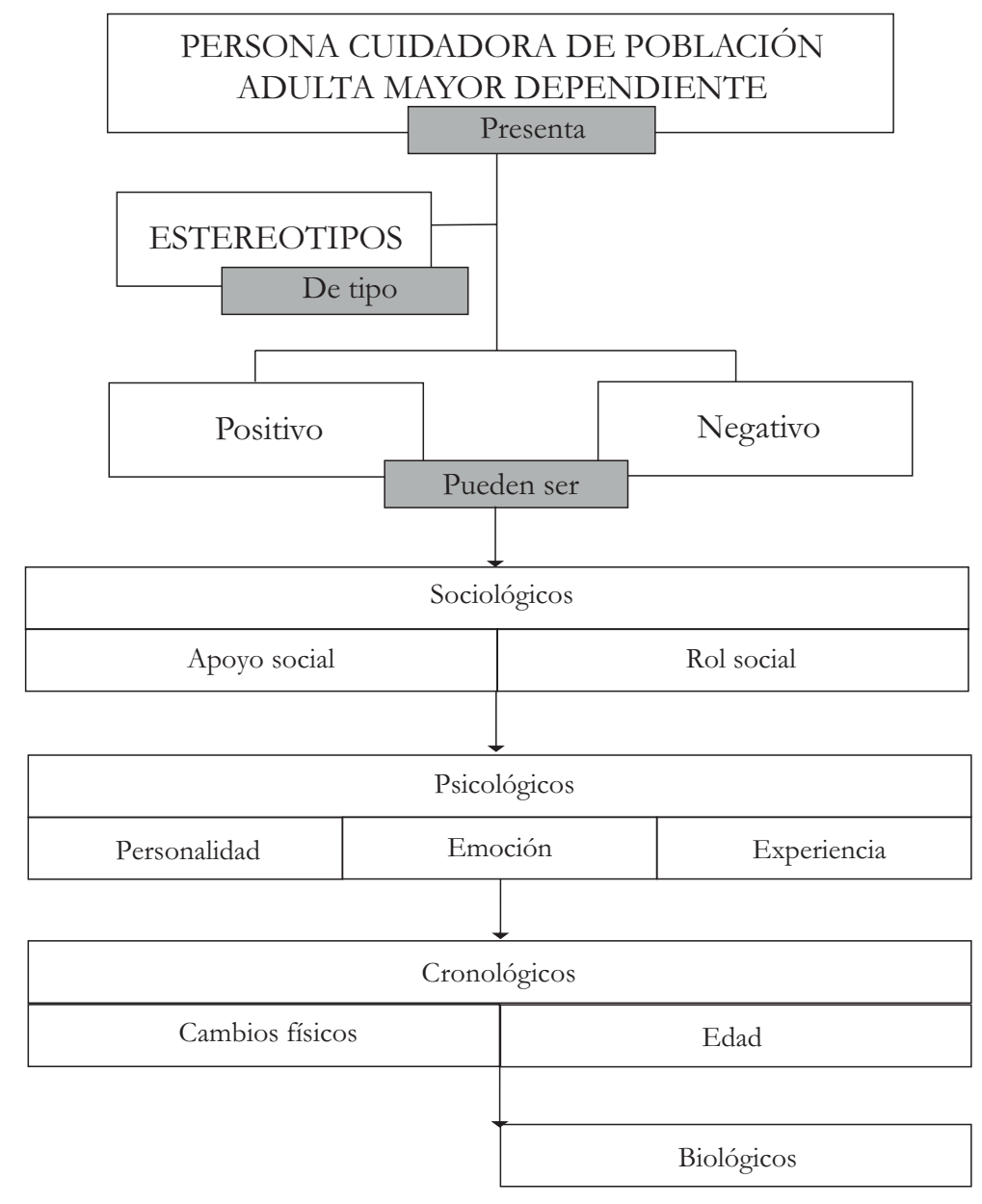

Figura 2. Esquema general de categorías: estereotipos en cuidadores 


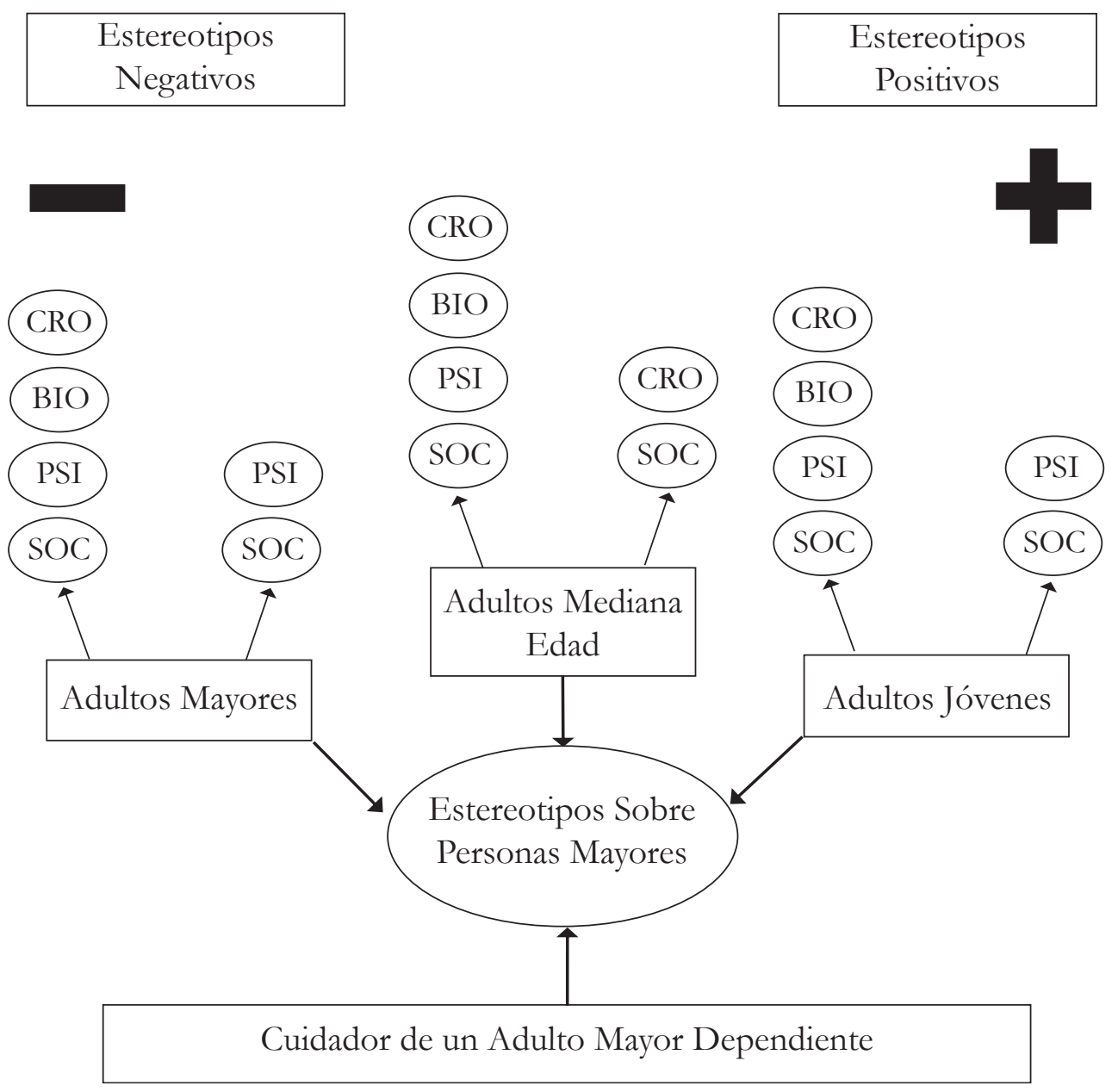

Figura 3. Esquema análisis de categorías por grupo etario

\section{Estereotipos positivos}

Las personas cuidadoras de los tres grupos etarios consideran dentro de los estereotipos sociológicos que, las PAM tienen herramientas para relacionarse en diferentes contextos. Por otro lado, los estereotipos psicológicos se basan en la experiencia y la sabiduría desde la percepción de los jóvenes y mayores. No se manifestó ningún estereotipo positivo biológico y los estereotipos cronológicos son presentados únicamente por adultos medios. Estos estereotipos incluyen características de personalidad positiva, un rol social especial y capacidad de mantenimiento o recuperación ante los cambios físicos con el paso del tiempo, lo que, desde el discurso de los participantes, se sustenta principalmente por la experiencia que han adquirido las PAM.

Estereotipos Psicológicos. Las personas cuidadoras jóvenes y adultas mayores presentan estereotipos positivos de tipo psicológico relacionados con la personalidad de las PAM. Los jóvenes afirman que "La personalidad de un adulto mayor puede ser más tranquila, más calmada", "Toman la adultez como pasiva, muy en calma"; las personas cuidadoras 
adultas mayores verbalizan que "Piensan diferente y actúan diferente por los años que han vivido", "Son los únicos que nos pueden dar un consejo sabio", "Son más inteligentes", "tienen mucho conocimiento".

Los jóvenes también refieren estereotipos psicológicos desde la experiencia del adulto mayor por los años vividos cuando comentan que "Son los únicos que nos enseñan la vida", "Tienen mucha más sabiduría para enseñarnos a los demás", "La experiencia y los años hace que las personas adultas sean más sabias", "Saben orientar más", "Son los que hablan con propiedad".

Estereotipos Sociológicos. Todos los grupos etarios presentan estereotipos de tipo sociológico asociados al rol social de las PAM; cuando describen cómo interactúan o los ven desenvolverse en la sociedad, los jóvenes refieren que "reflejan más confianza", "son más confiables porque tuvieron una buena educación y buenos principios", "no se dejan llevar por las emociones".

Por su lado, los adultos de mediana edad verbalizan que las PAM "Se relacionan muy fácilmente con las demás personas", "Es más confiable el adulto mayor por la seriedad y la experiencia", "Ellos se integran entre sí, participan en muchas actividades", "La vida social del adulto mayor es en toda parte, es en la familia, en el trabajo, con sus amigos, con todos él es muy sociable" y las personas cuidadoras adultas mayores refieren que "uno le tiene más confianza a un mayor que a un joven", "me he sentido más apoyada por una abuela que por pelaos que no tienen experiencia", "son más confiables, ya saben de las situaciones por las que pasan las familias, los hogares, los hijos", "son autosuficientes, tanto económicamente, como anímicamente".

Estereotipos Cronológicos. Solo los adultos de mediana edad presentan estereotipos positivos de tipo cronológico asociados a los cambios físicos, al referir que las PAM con el paso del tiempo pueden mantenerse o recuperarse porque "son personas que saben conservar su vejez, ya están preparados para esa edad", que ante los cambios "hay que seguir adelante y tratar de ser él mismo", "van a tener una buena autoestima", "se valoran".

\section{Estereotipos negativos}

Se reconocen más estereotipos negativos en todos los grupos etarios de cuidadores, los estereotipos cronológicos se sustentan con creencias erradas del momento en que una persona empieza a considerarse adulta mayor y ello implica una proximidad con la muerte. Esta categoría también se sustenta desde los cambios, principalmente físicos; los cuáles se consideran efectos ineludibles del paso del tiempo y que tienen que ver con pérdida, deterioro y dependencia, dificultades para desarrollar actividades y necesidad de cuidado.

Los estereotipos biológicos son los de mayor manifestación, se destaca una visión de pérdida por parte de todos los grupos etarios, necesidad de ayuda por parte de otros, y experimentación de temores como efecto ante dichos cambios; los tres grupos etarios conciben el envejecimiento como un proceso cargado de achaques físicos, con abundancia de enfermedades y con cercanía a la muerte.

Estereotipos psicológicos. Los cuidadores hacen énfasis en los cambios de la personalidad y en la emoción de las PAM para destacar el declive de sus recursos psicológicos y sensoriales, 
la vulnerabilidad, tristeza, soledad, y agresividad. Finalmente, los estereotipos sociológicos están marcados por una tendencia de limitación por parte de la PAM para interactuar, desvinculación e inutilidad. Lo que lo hace necesitar a la familia fundamentalmente para su cuidado, aunque destacan que el cuidado no es adecuado, los excluyen y prefieren asignar dicho cuidado a otros, o internarlos en asilos.

Estereotipos cronológicos. Las personas cuidadoras jóvenes, en mediana edad y adultos mayores presentan estereotipos negativos de tipo cronológico asociado a los cambios físicos. Los jóvenes refieren que "Se sentirán tristes porque su cuerpo no responde como si tuviera 20" igualmente los adultos de mediana edad verbalizan que, "ya no es capaz de hacer las cosas" y los cuidadores adultos mayores indican que "ya se ven muy viejitos y feitos", "pierde las capacidades", "ya uno no sirve para nada", "es obligatorio que necesitan ayuda".

Los estereotipos de tipo cronológico asociados con la edad son presentados por cuidadores jóvenes y en mediana edad, al indicar que una persona es vieja a los 55 años y que "La muerte empieza cuando ya se ha vivido todo", "a esa edad ya uno tiene que morirse porque ya cumplió con su meta, ya para qué más tiempo", "Causa miedo porque se aproxima la muerte", "ya tiene que morar para descansar".

Estereotipos biológicos. Los cuidadores jóvenes verbalizan que las PAM presentan "enfermedades", "Poca movilidad", "la masa corporal se disminuye muchísimo, entonces se vuelven flaquitos, mayor riesgo de fracturas, pérdida de los reflejos, dolores musculares", "Pérdida de la vista, pérdida de la actividad física", "lentitud para hacer las cosas, para comer", "No controlan sus esfínteres".

Los cuidadores de mediana edad afirman que "Tiene más debilidad al caminar porque con cualquier cosa se tropiezan", "Dolores de cabeza por nada, cansancio, fatiga, la parte del equilibrio" y los cuidadores adultos mayores "Tienen muchas enfermedades, se ven débiles", "Tienen más dolencias en el cuerpo" "Ya no pueden tener el movimiento motriz de hace años porque ya las células, ya se han deteriorado".

Estereotipos psicológicos. En relación con la emoción del adulto mayor, los cuidadores jóvenes presentan verbalizaciones como "la depresión porque tiene que depender de otra persona" "todos los adultos mayores pueden llegar a ser depresivos" que pueden experimentar "sentimientos de minusvalía, incapaces de lograr hacer las actividades lo que genera que se sientan reprimidos, dependientes, como yo no sirvo para la familia, ni para los cuidadores".

Las personas cuidadoras de mediana edad indican que "hay más depresión, soledad, tristeza", "la sensibilidad porque volveremos a ser como niños, vamos a tener chocheras", "el adulto mayor no tiene el mismo cariño que tenía antes... lo dejan, lo abandonan"; finalmente las personas cuidadoras adultas mayores piensan que "sienten que son una carga para la familia o para alguien, ya no es autosuficiente".

La segunda subcategoría emergente de los estereotipos negativos psicológicos es personalidad, donde los cuidadores jóvenes usan expresiones como "se puede tornar un poco malgeniado, estresado", "vuelven a ser como niños, y empiezan a ser un poquito más cansones"; los cuidadores de mediana edad indican que "son más cansones, molestan, ya nada les gusta, nada los tiene contentos" "necesitan de mucho afecto... son 
muy vulnerables". Las PAM entrevistadas refieren que "se vuelven soberbias, agresivas, perezosas, no quieren hacer nada" "se vuelven aislados, ya no quiere hablar, ya no sonríe".

Finalmente, en la categoría experiencia los adultos jóvenes y medios reportaron que el paso del tiempo no hace a las personas más sabias, "porque van perdiendo la memoria, la visión" "con el paso del tiempo van perdiendo la memoria y ya no se acuerdan de lo que han pasado".

Estereotipos sociológicos. Los estereotipos sociológicos son descritos por los cuidadores desde el rol social del adulto mayor; las personas cuidadoras jóvenes piensan que estos "Se dedican es a orar", "se ven muy limitados", "No quieren llegar a esa etapa donde todo el mundo los considera viejos", "No expresan sus emociones o cosas que piensan a las personas más cercanas", "su entorno se convierte la familia nada más".

Por otro lado los cuidadores de mediana edad refieren que "Tienen una visa social aislada, la misma sociedad se encarga", "no son confiables porque hay más que todo hombres que se devuelven a la juventud y abusan mucho de las niñas", "en la actualidad hay más abusadores adultos mayores", "la vida social de un adulto es muy aburrida", "ya nadie los toma en cuenta pues empiezan a sentirse inútiles para la sociedad" y las personas cuidadoras adultas mayores verbalizan que "Son muy difíciles para tratar", "Les cuesta mucho relacionarse", "Pocos se integran", "Permanecen encerrados, no son sociables", "la gente se burla de los viejitos, los empujan".

Adicionalmente, los estereotipos sociológicos son relacionados con el apoyo familiar, los cuidadores jóvenes piensan que "La mayoría de familias excluyen al adulto mayor", "si no tienen apoyo caen en depresión", "en la mayoría de familias excluyen al adulto mayor", "Los consideran como si fueran un estorbo" los cuidadores de mediana edad indican que "Los cansan y la decisión es dejarlos en un asilo para que lo cuide otro", "las redes de apoyo los tienen bastante descuidados", "no les ponen la atención que requieren"; los cuidadores adultos mayores verbalizan que "Todos los adultos mayores van a terminar como en un asilo, en la calle, arrimados, de casa en casa, porque no los soportan o porque ya les fastidia".

\section{Discusión}

Las personas cuidadoras entrevistadas se caracterizan por ser de género femenino, lo que confirma la feminización del cuidado mencionado en algunos estudios (Cerquera, Matajira, \& Pabón, 2016; Espín, 2008); en su mayoría estos son cuidadores de tipo informal, lo que coincide con el planteamiento de Aparicio et al. (2008, citado en Pabón et al., 2014) quienes destacan que el rol de cuidador informal es más común que el rol de cuidador formal.

En cuanto a la cantidad de participantes por grupo etario para el estudio, se determinó en función de la saturación de las categorías, tal como se recomienda en los estudios cualitativos (Hernández-Sampieri et al., 2014). Se resalta que incluir personas cuidadoras jóvenes, de mediana edad y adultas mayores permitió evidenciar que todos los grupos etarios presentan estereotipos, lo que es acorde con los hallazgos de otros estudios que analizan el fenómeno en diferentes grupos etarios (Lasagni et al., 2012; Cerquera et al., 2010; Chaparro et al., 2014). 
Dentro de las expresiones con connotación negativa para referirse a las PAM, se identificaron "incapacidad de valerse por sí mismos" "estado de enfermedad" "achaques físicos" "con cercanía a la muerte" "depresivos", entre otros. Lo que es coincidente con la afirmación de Callís-Fernández (2011), quien indica que las PAM son vistas como un grupo marginal y la imagen general está basada en términos inútiles e improductivos. Asimismo, Arnold-Cathalifaud et al. (2007) identifican dentro de las expresiones que usan jóvenes Chilenos para referirse a los AM, el ser desvalorizados socialmente, marginados, dependientes e improductivos.

Lo mencionado anteriormente, coincide con los estereotipos negativos de tipo sociológico de este estudio, en los que se destaca precisamente la desvinculación social, la inutilidad e improductividad de las PAM (Carbajo, 2009; Fernández \& Antón, 2018). Sin embargo, en dicha categoría, este estudio también contó con la presencia de estereotipos positivos marcados, siendo el único que se presentó en los tres grupos etarios. Así se asigna un rol especial en la sociedad a las PAM, por su conocimiento y sabiduría, por sus buenas conductas y manejo emocional, los cuáles se dan por la experiencia que poseen.

En las categorías psicológica y sociológica de los estereotipos negativos, se evidencia que los tres grupos etarios asocian a la vejez y al envejecimiento con cambios emocionales importantes, donde también hay pérdida de sus facultades cognitivas y en sus características de personalidad siendo más irritantes, estresantes o malgeniados, tal como lo plantean Domínguez (1983, citado en Carbajo, 2009). Además, encuentra relación con las categorías motivación y personalidad planteadas por Lasagni et al. (2012), quién afirma que se continúan percibiendo a los gerontes en términos de carencias afectivas, falta de intereses vitales y capacidad disminuida para desempeñar una actividad laboral, personas con rigidez mental, problemas de labilidad emocional y conductas que demarcan un debilitamiento del estatus adulto.

Lo anterior, también es apoyado con los hallazgos de Cerquera et al. (2010) en diferentes grupos poblacionales, Arnold-Cathalifaud et al. (2007) en jóvenes, Arístizabal et al. (2009) en estudiantes de carreras de la salud y Franco et al. (2010) en personal de salud de un hospital. De la presencia de los estereotipos psicológicos y sociológicos, este estudio resalta una interacción constante en las verbalizaciones, pues para los cuidadores la pérdida de recursos psicológicos, cognitivos, de personalidad, pero principalmente emocionales, son los que generan limitaciones en el rol social de las PAM, necesidad de apoyo social y familiar; aunque perciban que dicho apoyo sea escaso.

En estereotipos negativos de la categoría cronológica, las personas cuidadoras jóvenes y de mediana edad principalmente, consideran que entre más años ha vivido una persona, es decir por llegar a la vejez o estar próximo a ella, genera la necesidad de descanso y de morir para descansar. Lo que a su vez se relaciona con los cambios o pérdidas que se dan únicamente por el paso del tiempo y que son negativos, de manera que al tener determinada edad "se pierden la movilidad y estabilidad" "ya no es capaz de hacer las cosas" o "no sirve para nada" y esto muestra coincidencia con otros hallazgos de Cerquera et al. (2010) y Lasagni et al. (2012). 
En la categoría biológica, categoría que presenta mayor manifestación entre las personas cuidadoras de los tres grupos etarios, se resalta la necesidad de ayuda por parte de otros y experimentación de temores debido al deterioro al que se puede llegar. Lo que es unánime con los hallazgos de Lasagni et al. (2012), ya que se evidenció una percepción negativa de la imagen hacia el adulto mayor sobre su vejez o el proceso de envejecimiento, aunque en su estudio el análisis se realiza desde la categoría salud, también se reportaba la percepción de deterioro y de la aparición de discapacidades. De igual manera en el estudio de Franco et al. (2010), pero con personal de salud de un hospital.

La presencia de los estereotipos negativos de tipo biológico, se soportan desde lo que afirman Dulcey y Uribe (2002), quienes señalan que hay una tendencia a pensar que la vejez y el envejecimiento implican una pérdida de las funciones físicas y psicológicas; así este estudio permite esclarecer que en esta muestra de cuidadores que interactúan con PAM dependientes, los estereotipos están marcados por una consideración médica de la vejez que destaca involución y senilidad. Se destaca también que las categorías cronológica y biológica presentan relación, ya que la pérdida y el deterioro que se genera desde lo biológico se asocia con el paso del tiempo, con llegar a la vejez, con el criterio de edad como determinante para envejecer.

Surge la hipótesis de que debido a la experiencia de cuidar PAM dependientes, los participantes asumen que el envejecimiento patológico, aquel en el que hay presencia importante de enfermedad y el que probablemente presenta la persona mayor a su cuidado, es el que define el proceso de todos los seres humanos. Desconociendo que existen otros tipos de envejecimiento como el normal y el exitoso, en el que se puede ser independiente e incluso tener un rendimiento excepcional en etapas avanzadas (Dulcey \& Uribe, 2002).

Lo anterior, se da en un contexto en el que existe un creciente número de familias donde uno de sus integrantes debe asumir el rol de cuidado o donde se debe invertir en una persona de apoyo que se haga cargo de la asistencia de la PAM (Dulcey \& Uribe, 2002). Esto hace vital seguir indagando acerca de las experiencias, creencias y significados en cuidadores, así como en la relación que existe entre dedicarse al cuidado, especialmente informal, ya que es el tipo de cuidador que predomina en los hallazgos; y presentar estereotipos de tipo biológico.

Respecto al desarrollo de las investigaciones en psicogerontología, Cerquera et al. (2016) afirman que es primario ampliar la perspectiva de los factores que influyen sobre la calidad del cuidado que ejercen los cuidadores formales e informales. Asimismo, Palmore (1999, citado en Agredo, 2010) afirma que la presencia de estereotipos puede asociarse a actitudes y conductas discriminatorias positivas o negativas, según sea el caso; generando la posibilidad de que quienes poseen estereotipos pongan en marcha conductas y actitudes edadistas hacía la población adulta mayor (Pabón \& Delgado, 2017).

Por consiguiente, desde los hallazgos, este estudio recomienda trabajar estrategias de prevención e intervención para los cuidadores de personas mayores, de manera que se evite una afectación en la atención que brindan o en el bienestar de los gerentes; pues ya se ha demostrado que la presencia de estereotipos en cuidadores formales si afecta el bienestar de las PAM (Castellano, 2014; Bustillo-López \& Fernández-Ballesteros, 2012). 
Aspectos adicionales de los estereotipos positivos, los cuales presentaron una menor manifestación, se relacionan con las de connotación positiva que usaron las personas cuidadoras para referirse a las PAM, tales como "experiencia" "inteligentes" "conocedores" "tranquilos", coincidente con lo planteado por Agredo (2010), Cerquera et al. (2012) y Sánchez y Triana (2009), quienes destacan dentro de estos estereotipos positivos, el concebir a los mayores como "felices" y "sabios"; no obstante, hay que clarificar que al ser éstos positivos no dejan de ser generalizaciones falsas que desdibujan a las PAM (Agredo, 2010).

Una categoría que se presenta en todos los tipos de estereotipos positivos es la experiencia, a pesar de que en el análisis se ubicó principalmente en los estereotipos de tipo psicológico, se mantiene asociada a los otros estereotipos positivos; ser tranquilo, estar feliz, saber afrontar, conocer, poder orientar es debido a la experiencia, según los participantes. Sin embargo, esto no evita que se presenten actitudes edadistas y discriminatorios. Castellano (2014) evidenció que una actitud asociada a estereotipos positivos es el paternalismo, lo que hace que se limite la autonomía de las PAM dependientes, esto genera menor actividad en ellos.

Este estudio plantea que el abordaje de los estereotipos es fundamental en los contextos de cuido de las PAM dependientes, las cuales necesitan un acompañamiento que promueva autonomía y calidad de vida. Además, donde se prevenga la fragilidad y se obtengan cuidados integrados, ya sea en el domicilio o en las instituciones (Regato et al., 2018).

Anexado a esto, se hace indispensable que todos los grupos etarios reciban dicho abordaje. Lasagni et al. (2012), por ejemplo, recomiendan redoblar esfuerzos para intensificar el trabajo en las generaciones más jóvenes y realzar los aspectos positivos de la vejez mediante distintas acciones; asimismo, Dulcey y Uribe (2002) desde el planteamiento de la perspectiva del ciclo vital, destaca que las intervenciones en una generación repercutirán en las siguientes, que el mayor beneficio de un grupo de edad puede derivarse de intervenciones previas en un grupo de edad anterior.

\section{Referencias}

Agredo, C. L. (2010). Estereotipos viejistas en ancianos: actualización de la estructura factorial y propiedades psicométricas de dos cuestionarios pioneros. International Journal of Psychology and Psychological Therapy, 10(2), 259-278. Recuperado de http:// www.ijpsy.com/volumen10/num2/261/estereotipos-viejistas-en-ancianosactualizaci-ES.pdf

Álvarez-Gayou, J. L. (2003). Cómo hacer investigación cualitativa: fundamentos y metodología. Paídos Iberica: Ciudad de México, México.

Aristizabal, N., Morales, A., Salas, B., \& Torres, A. (2009). Estereotipos negativos hacia los adultos mayores en estudiantes universitarios. Cuadernos Hispanoamericanos de psicología, 9(1), 35-44. Recuperado de http://m.uelbosque.edu.co/sites/default/ files/publicaciones/revistas/cuadernos_hispanoamericanos_psicologia/ volumen9_numero1/articulo_3.pdf

Arnold-Cathalifaud, M., Thumala, D., Urquiza, A., \& Ojeda, A. (2007). Old age from the view of chilean youth: exploratory study. Última década, 15(27), 75-91. doi: 10.4067/S0718-22362007000200005 
Ballesteros, S. (2007). Envejecimiento Saludable: Aspectos, biológicos, psicológicos y sociales. Recuperado de http://www.uhu.es/auladelaexperiencia/documentos/1516/ AEXPERIENCIA-EnvejecimientoSaludable_Aspectos-BiologicosPsicologos[Ballesteros2007].pdf

Bustillo-López, A. \& Fernández-Ballesteros, R. (2012). Efecto de los estereótipos acerca de la vejez em la atención a adultos mayores. Salud Pública de México, 54(2). Recuperado de http://www.scielo.org.mx/scielo.php?script=sci_arttext\&pid $=$ S0036-36342012000200003

Callís-Fernández, S. (2011). Autoimagen del adulto mayor. Ciencia en su PC, (2), 30- 44. Recuperado de http://www.redalyc.org/pdf/1813/181322257004.pdf

Carbajo, M. D. (2009). Mitos y estereotipos sobre la vejez. Propuesta de una concepción realista y tolerante. Revista de la Facultad de Educación de Albacete, (24), 87-96. Recuperado de Dialnet-MitosYEstereotiposSobreLaVejezPropuestaDeUnaCon cep-3282988.pdf

Castellano, C. (2014). Análisis de la relación entre las actitudes hacía la vejez y el envejecimiento y los índices de bienestar en una muestra de personas mayores. Revista Española de Geriatría y Gerontología, 49(3), 108-114. doi: 10.1016/j. regg.2013.06.001

Cerquera, A. M., Álvarez, J. L., \& Saavedra, A. C. (2010). Identificación de estereotipos y prejuicios hacia la vejez presentes en una comunidad educativa de floridablanca. Psychologia. Avances de la disciplina, 4(1), 73-87. doi: 10.21500/19002386.1160

Cerquera Córdoba, A. M., Matajira Camacho, J., \& Pabón Poches, D. K. (2016). Caracterización de una muestra de cuidadores formales de pacientes con trastorno neurocognitivo mayor en Bucaramanga. Revista Virtual Universidad Católica del Norte, 47, 4-19. Recuperado de http:/ / revistavirtual.ucn.edu.co/index. $\mathrm{php} /$ RevistaUCN/article/view/739/1265

Cerquera, A. M., Meléndez, C., \& Villabona, C. (2012). Identificación de los estereotipos sobre envejecimiento femenino, presentes en un grupo de mujeres jóvenes. Pensamiento Psicológico, 10(1), 77-88. Recuperado de http:/ /www.scielo.org.co/scielo. php?script=sci_arttext\&pid=S1657-89612012000100006\&lng=en\&tlng=es

Cerquera, A., Pabón, D., Granados, F., \& Galvis, M. (2016). Sobrecarga en cuidadores informales de pacientes con Alzheimer y la relación con su ingreso salarial. Psicogente, 19(36), 240-251. doi: 10.17081/psico.19.36.1295

Chaparro, L., Sánchez, B., \& Carrillo, G. M. (2014). Encuesta de caracterización del cuidado de la diada cuidador familiar - persona con enfermedad crónica. Revista de ciencia y cuidado, 11(2), 31-45. Recuperado de https:// revistas.ufps.edu.co/index. $\mathrm{php} /$ cienciaycuidado/article/view/196/205

Dulcey, E. \& Uribe, C. (2002). Psicologia del ciclo vital: Hacia una visión comprehensiva de la vida. Revista Latinoamericana de Psicología, 34(1-2), 17-27. Recuperado de http://www.redalyc.org/pdf/805/80534202.pdf 
Espín, A.M. (2008). Caracterización psicosocial de cuidadores informales de adultos mayores con demencia. Revista Cubana de Salud Pública, 34(3), 1-13. Recuperado de: http:/ / scielo.sld.cu/scielo.php?script=sci_arttext\&pid=S0864-34662008000300008

Fernández, Y. \& Antón, M. (2018). Estereotipos de las personas mayores y de género en la prensa digital: estudio empírico desde la teoría del Framing. Prisma Social: Revista de Ciencias Sociales, 21 (2), 316-337. Recuperado de http:// revistaprismasocial.es/article/view/2443

Flóres, E., Rivas, E., \& Seguel, F. (2012). Nivel de sobrecarga en el desempeño eel rol del cuidador familiar de adulto mayor con dependencia severa. Ciencia y Enfermeria, 28(1), 29-41. doi: 10.4067/S0717-95532012000100004

Franco, M., Villareal, E., Vargas, E., Martínez, 1., \& Galicia, L. (2010). Estereotipos negativos de la vejez en personal de salud de un Hospital de la Ciudad de Querétaro, México. Revista Medica de Chile, 138(8), 988-993. Recuperado de https://scielo.conicyt.cl/ $\mathrm{pdf} / \mathrm{rmc} / \mathrm{v} 138 \mathrm{n} 8 / \operatorname{art} 07 . \mathrm{pdf}$

Hernández, R., Fernández, C., \& Baptista, P. (2014). Metodología de la investigación. Mc Graw Hill Education: Ciudad de México, México.

Hoyl-Moreno, T. (2016). Teorías actuales del envejecimiento. ARS MÉDICA, Revista de ciencias médicas, 32(2). doi: 10.11565/arsmed.v32i2.258

Lasagni, V., Rodríguez, M., Bernal, R., Tuzzo, M., Heredia, D., Muñoz, L... Días, P. (2012). Estereotipos hacia la Vejez en Adultos Mayores y en Estudiantes Universitarios en diez países de América Latina. Red Latinoamericana de Gerontología. Recuperado de http://www.gerontologia.org/portal/ archivosUpload/INVESTIGACION_ESTEREOTIPOS_HACIA_LA_ VEJEZ_EN_AMERICA_LATINA_2012.pdf

Lavretsky, H. (2005). Stress and depression ininformal family caregivers of patients withAlzheimer's disease. AgingHealth,1(1),117-133.doi:10.2217/1745509X.1.1.117

Menéndez, S., Cuevas, A. M., Pérez, J., \& Lorence, B. (2016). Evaluación de los estereotipos negativos hacia la vejez en jóvenes. Revista Española de Geriatría y Gerontología, 51(6), 323-328. doi: 10.1016/j.regg.2015.12.003

Ministerio de Salud de Colombia. (2016). Protección social: Ciclo de vida. Recuperado de https://www.minsalud.gov.co/proteccionsocial/Paginas/cicloVida.aspx

Organización Mundial de la Salud. (2015). Informe Mundial sobre el envejecimiento y la salud. Recuperado de http://apps.who.int/iris/bitstream/ handle/10665/186466/9789240694873_spa.pdf;jsessionid=E91F1B7A934B8D 22E262857FA3110C6B? sequence $=1$

Organización Mundial de la Salud. (2018). Envejecimiento y salud. Recuperado de https:// www.who.int/es/news-room/fact-sheets/detail/envejecimiento-y-salud

Pabón-Poches, D. K. (2019). Riesgo de maltrato en personas adultas mayores: validación de contenido de una escala. Psicogente, 22(41), 1-27. doi: 10.17081/psico.22.41.3299 
Pabón, D. K. \& Delgado, J. A. (2017). Maltrato en la población adulta mayor: una revisión. Espacio abierto: cuaderno Venezolano de Sociología, 26(2), 245-267.

Pabón, D., Galvis, M., \& Cerquera, A. (2014). Diferencias en el apoyo social percibido entre cuidadores informales y formales de pacientes con demencia tipo Alzheimer. Psicología desde el Caribe, 31(1), 59-77. Recuperado de http://www.redalyc.org/ $\mathrm{html} / 213 / 21330429004 /$

Regato, P., Hernández, M., \& Acosta, M. (2018). La semfyc quiere potenciar la investigación en el área del envejecimiento. Atención primaria, 510(6), 321-322. doi: 10.1016/j. aprim.2018.05.001

Sánchez, B., Chaparro, L., \& Carrillo, G. M. (2016). La carga del cuidado en la enfermedad crónica en la díada cuidador familiar-receptor del cuidado. Investigación Enfermería: Imagen y Desarrollo, 18(2), 43-60. doi: 10.11144/Javeriana.ie18-2.ccec

Sánchez, C. \& Triana, M. (2009). Estereotipos negativos hacia la vejez y su relación con variables sociodemográficas en personas mayores de 65 años. Revista Española de Geriatría y Gerontología, 44(3), 124-129. doi: 10.1016/j.regg.2008.12.008

Sánchez, C., Trianes, M., \& Mena, M. (2009). Estereotipos negativos hacia la vejez y su relación con variables sociodemográficas en personas mayores de 65 años. Revista Española de Geriatría y Gerontología, 44(3), 124-129. doi: 10.1016/j.regg.2008.12.008

Vicente-Ruiz, M. A., De la Cruz, C., Morales, R. A., Martínez, C. M., \& Villareal M. A. (2014). Cansancio, cuidadores y repercusiones en cuidadores formales de adultos mayores con enfermedades crónico degenerativas. European Journal of Investigation in Health, 4(2), 151-160. doi: https://doi.org/10.30552/ejihpe.v4i2.66 